\title{
Effect of Electric Field on the Mobility of Carboxyl-Terminated Dendrimers
}

\author{
E. Seyrek, P. L. Dubin,* and G. R. Newkome ${ }^{\dagger}$ \\ Department of Chemistry, Indiana-Purdue University, 402 North Blackford Street, Indianapolis, Indiana \\ 46202, and Departments of Polymer Science and Chemistry, The University of Akron, Akron, Ohio 44325-4717
}

Received: December 24, 2003; In Final Form: April 28, 2004

\begin{abstract}
The effect of an applied electric field on the mobilities of carboxyl-terminated dendrimers has been measured by capillary electrophoresis at applied voltages $V$ ranging from 5 to $30 \mathrm{kV}$. The mobility increased with the field strength at moderate ionic strength, but varied inversely with $V$ at low ionic strength. These results did not arise from Joule heating or from electroosmotic flow effects. It is suggested that the remarkably high velocities of these dendrimers enables them to outstrip their ion atmospheres at high $V$, in a manner related to the increase in conductance at high field strength first described by Wien in 1927. At low ionic strength, the increase in the size of the ion atmosphere leads to increased frictional drag with increasing field strength.
\end{abstract}

\section{Introduction}

The theory of electrophoresis explains the motion of a charged particle (or central ion) and its surrounding ion atmosphere in an electric field. ${ }^{1-3}$ An important consideration is the asymmetry of the ion atmosphere, which can result from the constant motion of the central particle that displaces it from the center of its ionic atmosphere. This displacement arises because, in addition to the primary response of the central particle to the externally applied field, two other forces act on the system. These are (1) the relaxation force, the tendency of the central particle to retain its ion atmosphere when the development of an asymmetric ion cloud locates its center of charge behind that of the central ion; and (2) the electrophoretic (drag) force exerted on the central particle through hydrodynamic coupling with its counterions. Since both of these effects are opposite in direction to the primary force, they act to retard the central ion and decrease its mobility relative to what it would exhibit in the absence of its ionic atmosphere.

The drag and relaxation forces noted above are generally considered to be affected by the external field in the same manner as the primary force, and hence the observed particle mobility is not expected to depend on the magnitude of the electric field. ${ }^{2}$ Nonlinear electrophoretic behavior could however occur if the central ion were to outstrip its ion atmosphere by moving so rapidly, e.g., at very high electric fields, that the ion cloud does not have sufficient time to build up, or if the effective concentration of counterions near the central ion itself becomes field-dependent. Such a nonlinear electrophoretic effect has been referred to as the "Wien effect", ${ }^{4}$ although strictly speaking this refers to the observation of an increase in the conductivity of simple electrolytes with increasing electric field strength. ${ }^{4-7}$ While this effect is assumed to be a reflection of particle velocities large vis-à-vis ion atmosphere relaxation times, direct observations of this effect in mobility measurements have been generally considered impractical because theory and experiment both indicate that the electric fields necessary to induce observable Wien effects for small ions, on the order of $100 \mathrm{kV} /$ $\mathrm{cm},{ }^{8,9}$ would generally be expected to produce unacceptable

\footnotetext{
* To whom correspondence should be addressed.

$\dagger$ The University of Akron.
}

levels of Joule heating. Increases in the conductance of polyelectrolytes with increasing field strength have, however, been reported at electric field strengths an order of magnitude lower. ${ }^{9,10}$ These observations are supported by recent simulations of polyelectrolytes yielding nonlinear electrophoretic behavior at fields also on the order of $10 \mathrm{kV} / \mathrm{cm} .^{11}$

The outstripping of the ion atmosphere by the central ion could be observed by electrophoresis if the charge of the particle were so large relative to its size that the velocity required for escape from its ion atmosphere could be attained at relatively low field strength. Extraordinarily high charge-to-size ratios are feasible for carboxyl-terminated dendrimers ${ }^{12}$ which can possess, for "generation 5", almost 1000 ionizable carboxylic acid groups, along with a hydrodynamic radius $R_{\mathrm{h}}$ of less than 4 $\mathrm{nm}$, corresponding to structural surface charge densities of nearly $1 \mathrm{C} / \mathrm{m}^{2}$, i.e., in excess of those achievable by other small colloids, such as micelles or proteins. The measurement of mobility at high electric field is also facilitated by the use of capillary electrophoresis, which allows for dissipation of Joule heating by efficient thermostating of a small capillary.

Capillary electrophoresis has been used to measure the mobility of dendrimers, but no reports of an effect of applied voltage on mobility have appeared. Huang et al. ${ }^{13}$ studied the effect of $\mathrm{pH}$ and ionic strength on the mobility of carboxylterminated dendrimers. Their most dramatic observation was that the mobility of the second generation (G2) dendrimer ( $R_{\mathrm{h}}=1.7 \mathrm{~nm}$, 36 charges) was $15 \%$ larger than that of $\mathrm{G} 5$ $\left(R_{\mathrm{h}}=3.7 \mathrm{~nm}, 972\right.$ charges). This, coupled with the fact that surface potentials measured by $\mathrm{pH}$ titration were smaller for G5 despite its much large surface charge density, led these authors to conclude that counterion condensation suppressed both the surface potentials and $\zeta$-potentials of G5. Welch et al. ${ }^{14}$ reported extensively on the electrophoretic behavior of Astromol amine-containing dendrimers, and concluded that they could be properly modeled as charged spheres. While they did not report any field-strength dependence of mobility, they stated that dendrimers are in the right size range (order of nanometers) to maximize the magnitude of the electrophoretic relaxation effect. We therefore report here observations of the dependence of the mobility of surface carboxylated dendrimers (G2-G5) on the electric field strength as a function of ionic strength. 
TABLE 1: Characteristics of Carboxyl-Terminated Dendrimers $^{a}$

\begin{tabular}{|c|c|c|c|c|}
\hline generation & $\begin{array}{l}\text { no. of } \\
\text { terminal } \\
\mathrm{COOH}\end{array}$ & MW & $\begin{array}{l}\text { hydrodynamic } \\
\text { radius }(\mathrm{nm})\end{array}$ & $\begin{array}{l}\text { surface charge } \\
\text { density }\left(\mathrm{C} / \mathrm{m}^{2}\right)\end{array}$ \\
\hline 2 & 36 & 4092 & 1.7 & 0.16 \\
\hline 3 & 108 & 12345 & 2.4 & 0.24 \\
\hline 4 & 324 & 37102 & 3.3 & 0.38 \\
\hline 5 & 972 & 111373 & 3.7 & 0.94 \\
\hline
\end{tabular}

\section{Experimental Section}

Materials. Previously synthesized carboxylic acid terminated dendrimers \{(Z-cascade:methane[4]:(3-oxo-6-oxa-2-azaheptylidyne):(propanoic acids) $\}^{12}$ of generations $2-5$ were purified by dialysis with molecular weight cutoff equal to 1000 , followed by freeze-drying. ${ }^{13}$ Some physical characteristics of these dendrimers are given in Table 1.

Boric acid, $\mathrm{H}_{3} \mathrm{BO}_{3}$, purchased from Sigma Chemical Co. (St. Louis, MO), and $\mathrm{NaOH}$ from Mallinckrodt Inc. (Paris, KY) were used to prepare borate buffer for capillary electrophoresis (CE). $\mathrm{NaOH}$ solutions (1 and $0.1 \mathrm{~N}$ ) for capillary rinsing were from Fisher Scientific (Pittsburgh, PA). Mesityl oxide, used as the neutral marker for $\mathrm{CE}$, was from Aldrich Chemical Co. (Milwaukee, WI). All solutions were prepared with Milli-Q water (Millipore, Milford, MA).

Capillary Electrophoresis. Experiments were carried out on a Beckman P/ACE 5500 instrument (Beckman, Fullerton, CA) with UV detection at $214 \mathrm{~nm}$. Untreated fused silica capillaries (Restek Corp, Bellefonte, PA) with $50 \mu \mathrm{m}$ inner diameter were of $27 \mathrm{~cm}$ total length and $20 \mathrm{~cm}$ effective length (distance from the injection end to the detector). To ensure complete ionization of the dendrimer carboxyl groups, samples were prepared with boric acid buffer at $\mathrm{pH} 9$, at ionic strengths $0.1,0.05$, and 0.01 $\mathrm{M}$. The applied voltage ranged from 5 to $30 \mathrm{kV}$. The temperature was maintained at $25^{\circ} \mathrm{C}$. Mobilities were measured by reference to the neutral marker mesityl oxide. Sample injection was for 5 $\mathrm{s}$ following injection of neutral marker for $3 \mathrm{~s}$. Prior to each experiment, the capillary was rinsed for 30 min with $0.1 \mathrm{~N}$ $\mathrm{NaOH}$, for 30 min with Milli-Q water, and for 30 min with the run buffer. Between runs, the capillary was rinsed with $0.1 \mathrm{~N}$ $\mathrm{NaOH}$.

The electrophoretic mobility $\mu_{\mathrm{E}}$ was calculated from

$$
\mu_{\mathrm{E}}=\frac{v_{\mathrm{o}}-v_{\mathrm{s}}}{E}=\frac{l L}{V}\left(\frac{1}{t_{\mathrm{m}}}-\frac{1}{t_{\mathrm{s}}}\right)
$$

where $v_{\mathrm{o}}$ and $v_{\mathrm{s}}$ are the electroosmotic velocity and the solute velocity respectively, $E$ is the applied field strength, $l$ and $L$ are the effective and total length of the capillary, respectively, $V$ is the applied voltage, and $t_{\mathrm{m}}$ and $t_{\mathrm{s}}$ are the retention times of the neutral marker and the sample, respectively.

\section{Results and Discussion}

Electrophoretic mobilities of G2-G5 dendrimers were measured at $\mathrm{pH} 9$ and ionic strengths 0.1 and $0.01 \mathrm{M}$ at varying applied fields. Sample electropherograms for G5 at two ionic strengths are shown in Figure 1, for nominal field strengths from 5 to $30 \mathrm{kV}$ (voltages above $20 \mathrm{kV}$ could not be applied at 0.1 $\mathrm{M}$ salt due to high voltage leak error). The neutral marker is the peak aligned at zero mobility. Due to their high negative charge, dendrimers have high mobilities and hence elute later than the neutral marker.
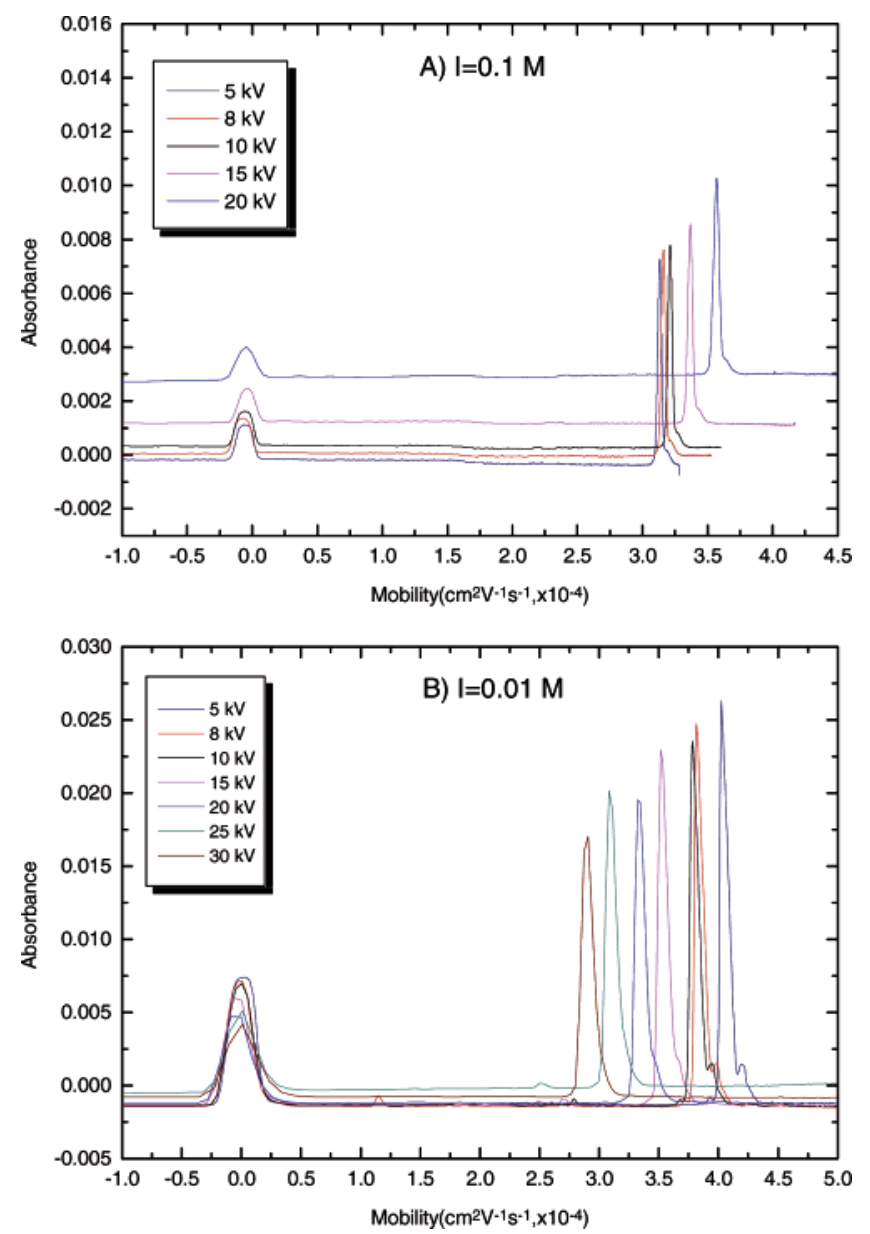

Figure 1. Sample electropherograms for G5 dendrimers at (A) 0.1 and (B) $0.01 \mathrm{M}$ ionic strengths, at varying applied voltages. $X$-axis is converted from time to mobility; the neutral marker is the peak at zero.

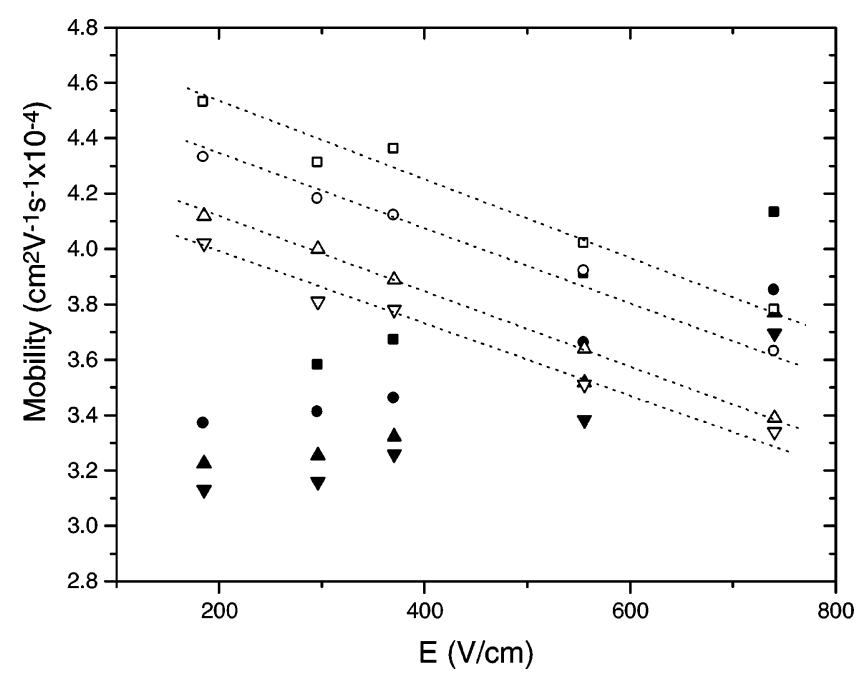

Figure 2. Electrophoretic mobility of dendrimers: (ם) G2, (•) G3, $(\boldsymbol{\wedge}) \mathrm{G} 4,(\boldsymbol{\nabla}) \mathrm{G} 5$. Filled and open symbols are at 0.1 and $0.01 \mathrm{M}$ buffer concentration, respectively. Lines are drawn to compare the magnitude of effects for different generations (see text).

The response of mobility to increasing voltage can be deduced from the shifts in the dendrimer peak position shown in Figure 1. As indicated by Figure 2, the dependence of mobility on field strength exhibits opposite tendencies at high and low ionic strengths. All other dendrimers, G2-G4 (electropherograms not shown), also showed the same behavior, i.e., increasing mobility with applied voltage at high salt, but inverse dependence at low 


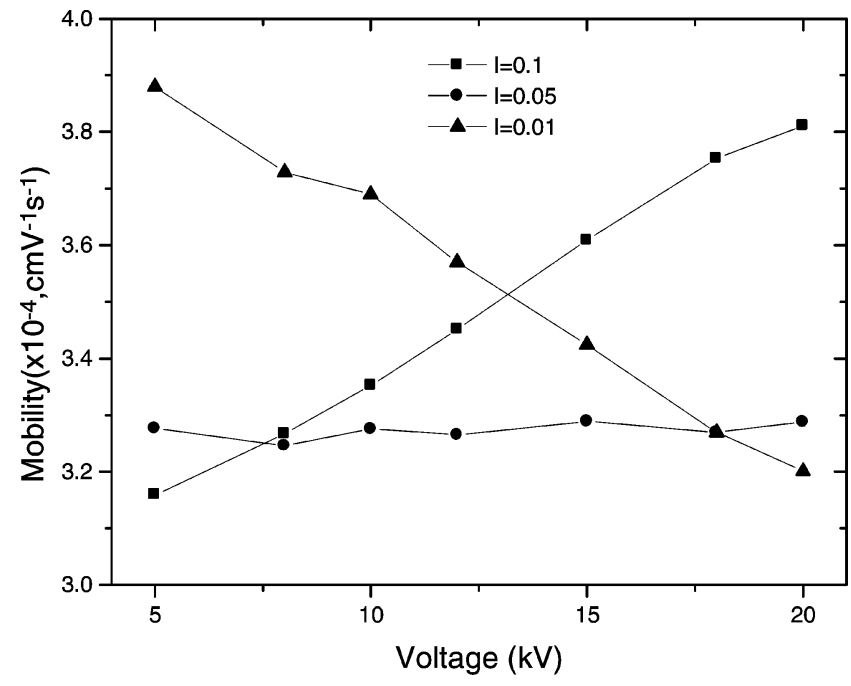

Figure 3. Electrophoretic mobility of G5 dendrimer at ionic strengths $0.1,0.05$, and $0.01 \mathrm{M}$ at different applied voltages.

salt. Under the typical conditions optimized for limited power dissipation, effective mobilities are generally considered to be independent of applied voltage. ${ }^{1,15}$ However, Figure 2 shows for dendrimers a strong dependence on applied electric field, with a significant role of ionic strength. The effect of ionic strength is continuous, and at an ionic strength of $0.05 \mathrm{M}$, i.e., intermediate between the cases of low and high salt shown in Figure 2, the mobility becomes field independent, as shown in Figure 3 for G5. Since the behaviors at low and high salt are opposite in direction, they arise either from a single phenomenon that changes monotonically with ionic strength, or from two different opposing effects that essentially cancel at intermediate salt concentration.

The increase in mobility with increasing applied voltage at $0.1 \mathrm{M}$ ionic strength suggests some phenomenon related to the

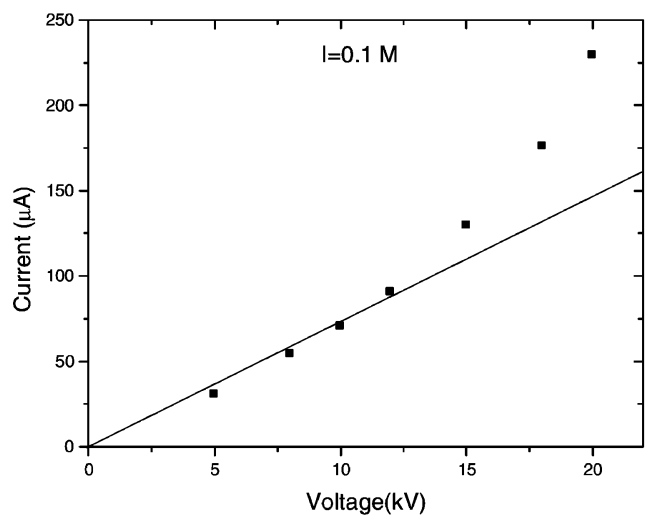

Wien effect, i.e., the ability of the central ion (here dendrimer) to outstrip its surrounding counterion cloud given a sufficiently high velocity. Prior to further discussion of this hypothesis, it is necessary to consider the influence of Joule heating. This is a significant problem for CE applications at high electric field, and is generally indicated by deviations from Ohm's law, i.e., nonlinearity in plots of current vs applied voltage. Ohm's law plots are given in Figure 4. At 0.1 and $0.05 \mathrm{M}$ ionic strength, nonlinearity is only observed above $\sim 15 \mathrm{kV}$, and at $0.01 \mathrm{M}$ only above $\sim 25 \mathrm{kV}$. Therefore, the voltage dependences of the mobility shown in Figure 3 must result from electrophoretic effects. This confirms a continuous change in the measured mobility, varying from a direct dependence on electric field at high salt to an inverse one at low salt.

To further verify that the observed nonlinearity arises from the motion of the particles, we calculated individually the electroosmosic flow (eof) shown in Figure 5. The eof velocity is obtained as the neutral marker migration velocity, which shows a linear response to increasing voltage at both ionic strengths, higher at low salt. However, unlike the effect of field strength on mobility, the dependence of eof on voltage is unidirectional regardless of ionic strength. The significant effect of buffer ionic strength on solute velocities in capillary electrophoresis is well-known, and it has been previously observed that electroosmotic flow varies inversely with buffer concentration. ${ }^{16,17}$ This demonstrates that the observed opposite behavior of mobility at high and low salt is not due to changes in the electroosmotic flow used to calculate the mobility.

Simulations, and to a lesser extent experimentation, have shown that dendrimers exhibit some conformational flexibility. ${ }^{19}$ At low $I$, the interaction of the unscreened dendrimer charges with the electric field might conceivably distort the dendrimer to a nonspherical shape with a higher friction coefficient, and this in principle could account for the decrease in mobility at low $I$. However, the conformational flexibility expected for

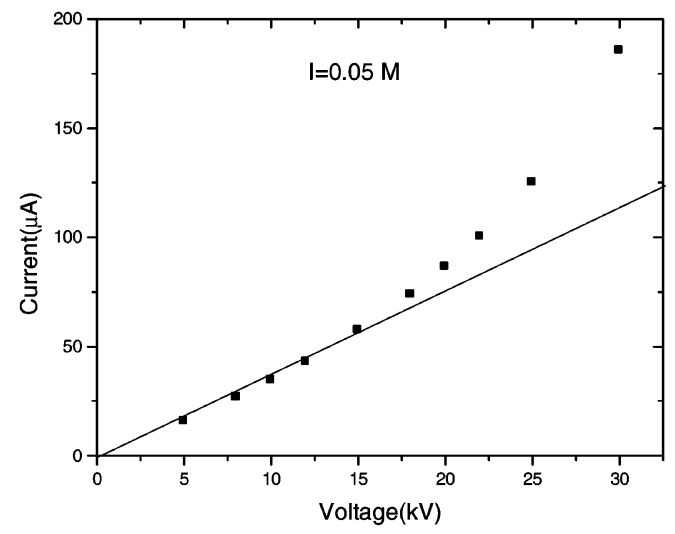

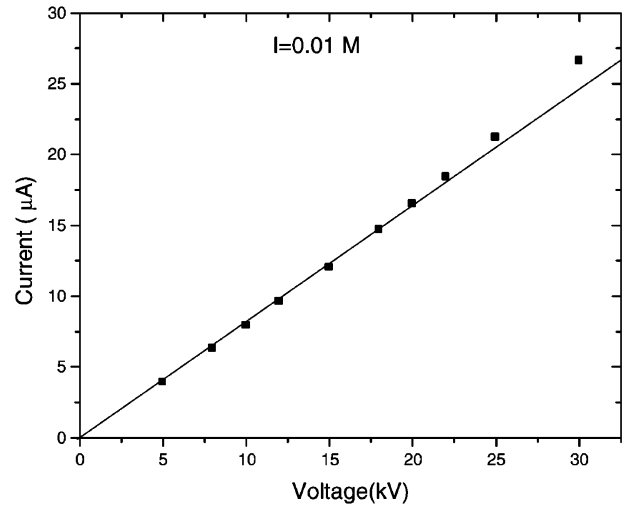

Figure 4. Ohm's law plots for the experiments corresponding to the data in Figure 3. 


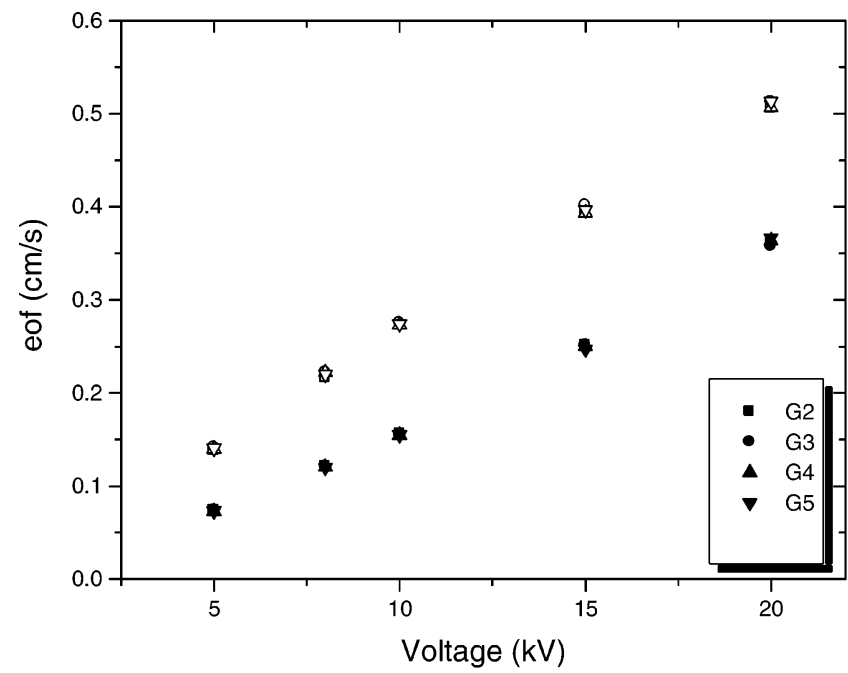

Figure 5. Voltage dependence of electroosmotic flow velocity. Closed symbols are $I=0.1 \mathrm{M}$, and open symbols represent $I=0.01 \mathrm{M}$, corresponding to the symbols labeled in the figure.

dendrimers of low distal congestion, e.g., low-generation PAMAM dendrimers, is unlikely in the present case, particularly for G5, with nearly 1000 fully charged, hence intramolecularly repulsive headgroups. Such effects might be more plausible for the lower generation dendrimers, but as displayed by dotted lines of Figure 2, the magnitude of the field effect at low salt is similar for all samples so that the observed behavior cannot be attributed to such conformational changes, but presumably arise from the electrophoretic effects exerted on the particle by the ionic cloud.

Apart from the field effects, we note that the mobility increases with decreasing generation number $\left(\mu_{\mathrm{G} 2}>\mu_{\mathrm{G} 3}>\mu_{\mathrm{G} 4}\right.$ $>\mu_{\mathrm{G} 5}$ ) despite the fact that the charge on the macroion is increasing significantly with dendrimer size (e.g., G5 has a net charge 27 times larger than G2). Huang et al. ${ }^{13}$ attributed this to counterion condensation on the macroion surface, reducing both its mobility and its surface potential (determined by potentiometric titration) when the charge per unit area exceeded some critical value. The accumulation of deuterated tetramethylammonium counterions around $\mathrm{G} 4$ at high $\mathrm{pH}$ was also directly observed by small angle neutron scattering contrast matching. ${ }^{18}$ However, Huang et al. did not explicitly consider the reduced friction coefficient of the smaller dendrimers, or the fact that their structures might allow for higher permeability to solvent, so questions remain about the extent to which the decreased mobility of higher generation dendrimers can be attributed to exclusively stronger counterion binding.

Counterions not condensed on the dendrimers but locally abundant clearly play a significant role inasmuch as the velocity of a macroion is determined not solely by the external applied field but also by the forces exerted by its surrounding ionic cloud. The size of this ionic cloud, which is roughly given by the Debye length, $\kappa^{-1}$, i.e., $\sim 1 \mathrm{~nm}$ at $0.1 \mathrm{M}$ and $\sim 3 \mathrm{~nm}$ at 0.01 M, should exert a significant influence on the extent of its effect. The abolition of this retardation when dendrimer velocity is so high that it escapes this ion cloud is our explanation of the effects at high salt, fundamentally consistent with the argument underlying the Wien effect. ${ }^{2,4}$ Conversely, at low $I\left(\right.$ large $\kappa^{-1}$ ), one might argue that insufficient time is available for this to occur. In other words, as long as the macroion retains its ion atmosphere, which is large at low $I$, retardation effects increase with its velocity, but at high salt $\left(\right.$ low $\left.\kappa^{-1}\right)$ the ability of the macroion to outstrip its ion atmosphere leads to the reduction of retardation effects and the increase in mobility with field strength. However, the time required for the central ion to travel the distance $\kappa^{-1}$ must be compared to the relaxation time of the ion atmosphere, defined ${ }^{2}$ as $\tau$, the time required for the small ions to diffuse the same distance $\kappa^{-1}$. Since $\tau$ is also large for low $I$, the preceding argument is simplistic. At the present time, the reason for the inversion of the salt effect at low $I$ is not clear.

\section{Conclusions}

Some of the results reported here are consistent with the conceptual foundation of the Wien effect, and these may not have been extensively noticed hitherto because the achievement of very high particle velocities without Joule heating is particularly favored by the unique combination of dendrimers and CE. While the retardation forces clearly depend on the ionic strength through the size of the ionic atmosphere, the electric field dependence of these forces cannot be resolved with existing theories based on rather simple models. However, even absent such explanations, one may still expect that the retardation forces should depend on the friction coefficient of the ions in the ionic cloud. Borate, for example, a relatively large ion, might enhance the magnitude of the observed effects. A more definitive test might be possible with dendrimer counterions of variable size, e.g., $\mathrm{Na}^{+}, \mathrm{Li}^{+},\left(\mathrm{Me}_{4} \mathrm{~N}\right)^{+}$, and $\left(n-\mathrm{Bu}_{4} \mathrm{~N}\right)^{+}$(in order of increasing expected size).

Acknowledgment. Support is acknowledged from NSF Grants CHE9987891, DMR0076068 (P.L.D.), and DMR9901393 (G.R.N.).

\section{References and Notes}

(1) Camilleri, P. Capillary Electrophoresis, Theory and Practice, 1st ed.; CRC Press: Boca Raton, FL, 1993.

(2) Bockris, J. O.; Reddy, A. K. N. Modern Electrochemistry, 2nd ed.; Plenum Press: New York, 1998

(3) O'Brien, R. W.; White, L. R. J. Chem. Soc., Faraday Trans. 2: Mol. Chem. Phys. 1978, 74, 1607-1626.

(4) Wien, M. Ann. Phys. (Berlin, Germany) 1927, 83, 327-361.

(5) Castellon, G. W. Physical Chemistry, 2nd ed.; Addison-Wesley: Reading, MA, 1966. 432.

(6) Onsager, L.; Liu, C. T. Z. Phys. Chem. (Leipzig) 1965, 228, 428-

(7) Onsager, L.; Kim, S. K. J. Phys. Chem. 1957, 61, 198-215.

(8) Harned, H. S. Physical Chemistry of Electrolytic Solutions; Reinhold: New York, 1950

(9) Grossman, P. D.; Soane, D. S. Anal. Chem. 1990, 62, 1592-1596.

(10) Bailey, F. E., Jr.; Patterson, A., Jr.; Fuoss, R. M. J. Am. Chem. Soc. 1952, 74, 1845-1846.

(11) Netz, R. R. J. Phys. Chem. B 2003, 107, 8208-8217.

(12) Newkome, G. R.; Moorefield, C. N.; Baker, G. R.; Potter, R. L.; Audoly, L.; Cooper, D.; Weis, C. D.; Morris, K.; Johnson, C. S., Jr.; Macromolecules 1993, 26, 2394-2396.

(13) Huang, Q. R.; Dubin, P. L.; Moorefield, C. N.; Newkome, G. R. J. Phys. Chem. B 2000, 104, 898-904.

(14) Welch, C. F.; Hoagland, D. A. Langmuir 2003, 19, 1082-1088.

(15) Weinberger, R. Practical Capillary Electrophoresis; Academic Press: San Diego, 1993. 231.

(16) Rasmussen, H. T.; McNair, H. M. J. Chromatogr. 1990, 516, $223-$

(17) Li, S. F. Y. Capillary Electrophoresis, Principles, Practice and Applications; Elsevier Science, Inc: Amsterdam, 1993.

(18) Huang, Q.; Dubin, P. L.; Lal, J. Unpublished results.

(19) Bosman, A. W.; Janssen, H. M.; Meijer, E. W. Chem. Rev. 1999, $99,1665-1688$ 